\title{
Parameters affecting pharyngeal response to genioglossus stimulation in sleep
} apnoea

\author{
Y. Dotan*, T. Golibroda\# , R. Oliven*, A. Netzer", L. Gaitini ${ }^{+}$, A. Toubi $^{\S}$ and A. Oliven*
}

ABSTRACT: Chronic stimulation of the hypoglossus nerve may provide a new treatment modality for obstructive sleep apnoea (OSA). In previous studies we observed large differences in response to stimulation of the genioglossus (GG). We hypothesised that both individual patient characteristics and the area of the GG stimulated are responsible for these differences.

In the present study, we compared the response to GG electrical stimulation at the anterior area (GGa-ES), which activates the whole GG and the posterior area (GGp-ES), which activates preferentially the longitudinal fibres. Studies were performed in 14 propofol-sedated OSA patients. The parameters evaluated included cephalometry, pressure-flow relationship and pharyngeal shape and compliance assessed by pharyngoscopy.

Compared with GGa-ES, GGp-ES resulted in significantly larger decreases in the critical value of end-expiratory pressure (Pcrit) (from $3.8 \pm 2.2$ to $2.9 \pm 3.3$ and $-2.0 \pm 3.9 \mathrm{cmH}_{2} \mathrm{O}$, respectively $(\mathrm{p}<\mathbf{0 . 0 0 1 )})$. Both tongue size and velopharyngeal shape (anteroposterior to lateral ratio) correlated significantly with the decrease in Pcrit during GGp-ES ( $R=0.53$ and -0.66 , respectively; $\mathbf{p}<0.05)$. In the patients with the larger tongue size $(n=7)$, the decrease in $P$ crit reached $8.0 \pm 2.2 \mathrm{cmH}_{2} \mathrm{O}$ during GGp-ES.

We conclude that directing stimulation to longitudinal fibres of the GG improves the flowmechanical effect. In addition, patients with large tongues and narrow pharynx tend to respond better to GGp-ES.

KEYWORDS: Critical pressure, electrical stimulation, genioglossus, sleep apnoea syndrome, velopharynx

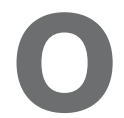
bstructive sleep apnoea (OSA) is a highly prevalent syndrome with multiple clinical implications [1]. It is estimated that OSA affects $4 \%$ of males and $2 \%$ of females, with continuously increasing incidence due to the obesity epidemic. The current treatment of OSA is the continuous positive airway pressure (CPAP) device, a simple yet very efficient modality, but unfortunately also uncomfortable, resulting in poor adherence. The obvious need for new treatment modalities is directed towards two lines of approach: anatomic (such as surgical procedures) and functional, i.e. electrical stimulation of the upper airway dilator muscles [2, 3]. The genioglossus (GG) muscle, which is the main tongue protrusor, has been shown to reduce pharyngeal resistance and collapsibility by far more than other upper airway dilator muscles [4, 5], and has become, therefore, the main target for functional stimulation for therapeutic purposes $[2,3]$.
In previous studies we found that GG contraction improves pharyngeal stability in OSA patients during sleep and anaesthesia [6,7], characterised by reduction of the pressure at which the pharynx collapses and occludes (the critical value of end-expiratory pressure, $P_{\text {crit}}$ ). However, large inter-individual differences in both the magnitude of decrease in $P_{\text {crit }}$ (i.e. improvement in stability) and enlargement of the pharynx were observed [7]. We hypothesised that both individual patient-characteristics and the mechanical function of the area of the GG where stimulation was applied may be responsible for these differences. As the GG fibres are arranged as a hand held fan, the anterior, vertical fibres cause primarily depression of the tongue, and only the dorsally oriented horizontal fibres protrude the tongue and enlarge the pharynx [8]. Accordingly, directing stimulation to the protrusive part of the GG is likely to improve the flow-mechanical

\section{AFFILIATIONS}

Depts of *Internal Medicine,

+Anesthesiology and,

${ }^{\S}$ Radiology, Bnai-Zion Medical Center,

"Dept of Otolaryngology, Rambam Medical Center, Haifa, and \# Ort-Braude College, Carmiel, Israel.

CORRESPONDENCE

Y. Dotan

Dept of Internal Medicine Bnai-Zion Medical Center 47 Golomb st.

Haifa 31048

Israel

E-mail: yanivdotan@gmail.com

Received:

Aug 062010

Accepted after revision:

Dec 082010

First published online:

Dec 222010 
response. In addition, we expected contraction of this muscle in subjects with a relatively large protrusive part of the GG to have a larger dilatory effect on the pharynx.

Identification of parameters that improve the response to functional stimulation of the tongue may help in selecting patients most appropriate for this approach as a treatment modality. Accordingly, the present study was designed to compare the effect of electrical stimulation of the anterior versus the posterior parts of the tongue on pharyngeal stability and size in OSA patients. In addition, we evaluated the relationship between individual relevant cephalometric characteristics of our patients and the response to electrical stimulation of the GG.

\section{METHODS \\ Subjects}

Letters were sent to all patients who had undertaken a full sleep study in the Technion Sleep Laboratory (Haifa, Israel) during the year prior to the present study and found to have an apnoea-hypopnoea index (AHI) $>20$, requesting them to participate in this research. Patients with any disease that could pose a risk during anaesthesia, including ischaemic heart disease, any lung disease, severe or uncontrolled hypertension and a body mass index (BMI) $>35 \mathrm{~kg} \cdot \mathrm{m}^{-2}$, as well as subjects with known side-effects to any previous anaesthesia, were excluded. All studies were performed in the respiratory research laboratory of Bnai Zion Medical Center (Haifa, Israel). The aims and potential risks of the study were explained and informed consent was obtained from all subjects. The study was approved by the Human Investigations Review Board of Bnai Zion Medical Center.

\section{Cephalometry}

Lateral cephalometric radiographs were performed with the subjects erect. The patients were instructed to gaze forward, holding their heads in a natural position with the jaw closed, and a digital head radiograph was taken. Exposure parameters were chosen to enable visualisation of both bony and soft tissue landmarks. Out of a large number of published cephalometric parameters [9-11], we chose for analysis seven variables considered to have the potential to affect the response to GG contraction (fig. 1). These parameters included mandible position in relation to the maxilla (angle between the nose base to the maxilla and the nose base to the chin), distance of the hyoid bone from the mandible and sagittal cross-sectional area of the velo-and oropharynx and soft palate. In addition, the sagittal cross-sectional area of the tongue, divided into the protrusor and depressor part of the GG (by drawing a line between the mid-point of mandibular insertion to the posterior edge of the hard palate) was also measured. In addition, the mouth-box was measured based on the TSUIKI et al. [11] description of the lower face cage with minor modifications. The mouth box was defined as the area enclosed by the front teeth, hard palate, lower margin of the mandible and the anterior boarder of the vertebral column. The cross-sectional size of the tongue was calculated also as a ratio of this area.

\section{Ultrasound}

In order to direct intramuscular electrode placement, submental soft tissue anatomy was examined using an ultrasound linear probe [12]. Coronal scans were obtained with the transducer directed vertically under the chin in the midline and axial scans obtained by turning the transducer $90^{\circ}$ from this position. During each scan, care was taken to minimise compression of the skin under the chin by the probe, and scanning sessions were recorded. The surface of the tongue, including the anterior wall of the pharynx, was visualised to assess the distance and direction for GG electrode placement, and Doppler was used to delineate the position of local blood vessels to prevent injury.

\section{Recording procedures}

Standard polysomnographic techniques, including submental surface electromyography (EMG), C3/O1 and C3/A2 electroencephalography (EEG), ECG and oxygen saturation measurement were employed to monitor the patient during anaesthesia and exclude arousal. Subjects breathed through a tight-fitting nasal mask and pneumotachometer, connected to a Validyne $\pm 2 \mathrm{cmH}_{2} \mathrm{O}$ pressure transducer (Los Angeles, CA, USA), with the mouth carefully and tightly sealed. The pneumotachometer was connected to a digitised variable pressure source at the inflow port, enabling variation of nasal pressure $\left(P_{n}\right)$ in the range of $20--10 \mathrm{cmH}_{2} \mathrm{O}$. $P$ n was monitored with a catheter connected to a side port of the mask. Intrathoracic pressure was measured with an oesophageal balloon catheter (Ackrad Laboratories, Cranford, NJ, USA), and used to identify flow limitation, as well as to distinguish between inspiration and expiration during complete apnoeas. Analogue-to-digital acquisition of all parameters was performed at $1,000 \mathrm{~Hz}$ for monitoring and data storage on a digital polygraphic data acquisition system (LabVIEW; National Instruments, Austin, TX, USA).

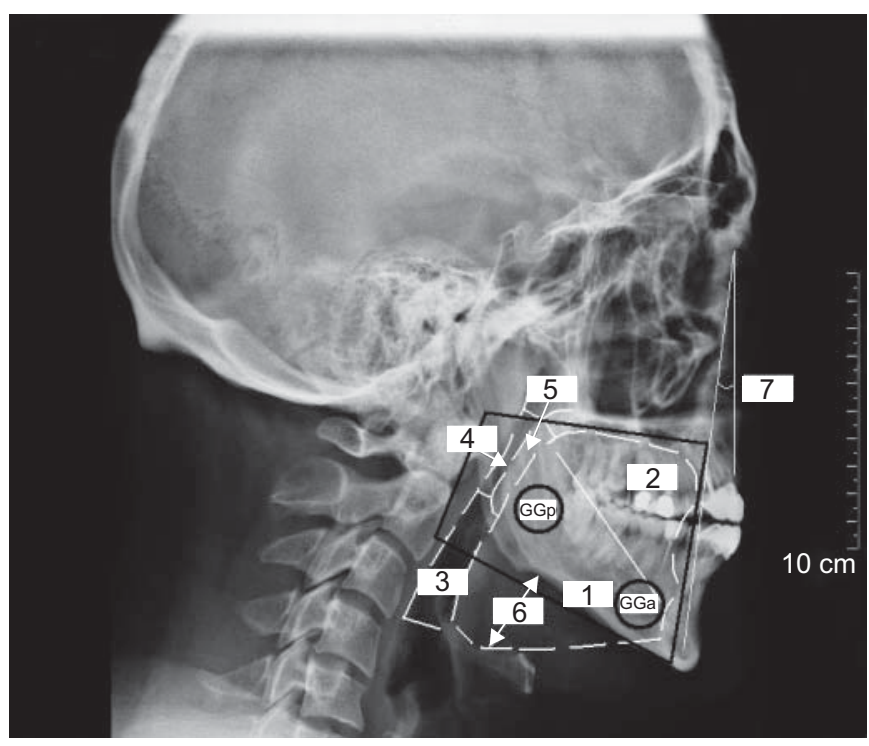

FIGURE 1. Cephalometric parameters: 1) The genioglossus (GG) part that advances the tongue; 2) the GG part that depresses and draws down the tongue; 3) oropharynx; 4) velopharynx; 5) soft palate; 6) distance between the hyoid bone and the mandible; and 7) angle of the chin-nose base-maxilla. The heavy black quadrangle delineates the mouth-box. The circles indicate the areas where the electrodes were positioned at the posterior (GGp) and anterior (GGa) sites of stimulation. 


\section{Anaesthesia}

Propofol anaesthesia was delivered by an anaesthesiologist using a loading dose of $2.5 \mathrm{mg} \cdot \mathrm{kg}^{-1}$ body weight and continuous drip of $6-12 \mathrm{mg} \cdot \mathrm{kg}^{-1}$ body weight per $\mathrm{h}$. Using positive levels of $P_{n}$ (i.e. CPAP) that enabled breathing without flow limitation, patients were maintained under stable anaesthesia that eliminated any reaction to pain and electrical stimulation, while maintaining adequate ventilation, as monitored by the pneumotachometer and pulse oximetry.

\section{Pharyngoscopy}

A flexible fiberoptic endoscope (Olympus BF-3C40; Olympus, Tokyo, Japan; with outside diameter $3.3 \mathrm{~mm}$ ) was inserted through an adequately sealed port in the nose mask and positioned above the site of collapse of the pharynx. The image was recorded on videotape, accompanied by audio explanations.

\section{Electrical stimulation}

GG stimulation was applied via teflon-coated $0.2 \mathrm{~mm}$ diameter hook wire electrodes with bared $0.13 \mathrm{~mm}$ diameter ends. The anterior electrodes were inserted sublingually, trans-mucosally, 10-15 mm deep into the anterior retromandibular body of the GG, as previously described [6,7]. The posterior electrodes were inserted transcutaneously through the submandibular area, directed by ultrasound guidance and the lateral head radiograph for angle and depth of insertion, to be positioned $2-3 \mathrm{~cm}$ ventral to the posterior surface of the tongue, at the level of the lower border of the soft palate (fig. 1). Four to six electrodes were inserted into the GG in each subject. Using a neuromuscular stimulator (Dynex III; Medtronic, Inc., Minneapolis, MN, USA), $40 \mathrm{~Hz}$ bursts of 6-8 s, with biphasic pulses of 100-ms width, were applied. Pharyngoscopic observation enabled selection of the electrodes and stimulation intensity that provided the best pharyngeal dilatory response. The intensity of stimulation was limited to levels that were well tolerated during wakefulness in previous and preliminary experiments.

\section{Experimental procedure}

After obtaining head radiographs and mouth base sonography, patients were prepared with EEG, submental EMG and venous access and placed in the supine position. Following induction of anaesthesia, CPAP was applied via a nose mask and raised to the level that abolished flow limitation (holding pressure). Thereafter, the endoscope, oesophageal balloon and both pairs of GG electrodes were positioned and the mouth was sealed. The primary site of collapse, determined visually during gradual reduction of $P_{n}$ and verified by the concomitant cessation of airflow, was at the level of the velopharynx in all our subjects. Therefore, the endoscope was placed above the area of velopharyngeal collapse. Thereafter, flow $/ P \mathrm{n}$ and pharyngeal area $/ P$ n relationships before and during GG stimulation were determined quasi-simultaneously, as previously described $[6,7]$. With the patient maintained at the holding pressure, $P \mathrm{n}$ was lowered randomly for a few breaths, encompassing four to six levels associated with inspiratory flow limitation and the level below which airflow ceased. At each $P \mathrm{n}$, after the fourth breath, stimulation was performed for two or three consecutive breaths, and, after additional two or three unstimulated breaths the $P_{n}$ was raised back to the holding pressure until stable baseline ventilation was observed. The same protocol was followed for each patient, once for posterior GG electrical stimulation (GGp-ES) and once for anterior GG electrical stimulation (GGa-ES), in random order. The same intensity of stimulation was used for both stimulation sites after determining the stimulation intensity that provided the best response in both sites.

\section{Data analysis}

The flow $/ P$ n relationship data were analysed using digital software and determined using least-squares linear regression. Maximal inspiratory flow ( $\left.V^{\prime} \max \right)$ was measured at the level at which inspiratory flow was maximal and plateaued while oesophageal pressure fell progressively, indicating the presence of flow limitation. This relationship was used to calculate the $P_{\text {crit }}$ as the $P$ n below which airflow became zero, as well as the flow $/ P_{n}$ slope. $\Delta P_{\text {crit }}$ (baseline $P_{\text {crit- }} P_{\text {crit }}$ during GG stimulation) was used to quantify the mechanical effect of GGa-ES and GGp-ES. Cephalometric areas of the digital head radiograph were outlined manually and calculated digitally using computer software. The video images of the pharyngeal lumen, recorded during evaluation of the flow $/ P$ n relationship before and during stimulation, were digitised and viewed, and single images from the end-expiratory pause were captured and stored. The respiratory frequency of all of the patients was relatively low (always $<20$ breaths $\cdot \mathrm{min}^{-1}$ ) due to the state of anaesthesia and high holding $P$ n used to prevent flow limitation, resulting in a sufficiently long end expiratory pause (always $>0.5 \mathrm{~s}$ ) during which intrapharyngeal pressure could become equal to $P$ n. The velopharyngeal cross-sectional area in each digitised frame was calculated digitally as described for the cephalometric areas. The oesophageal pressure tube, marked at regular levels, was used as a landmark, in addition to pharyngeal structures, to enable measurement of the area perpendicular to the pharyngeal axis and at the same distance from the endoscope before and during ES, and was used as a calibration reference for calculating the area in absolute units, as previously described [13]. The area/Pn relationship (i.e. pharyngeal compliance) was determined for the close-to-linear portion of this relationship only (i.e. below the $\mathrm{Pn}$ of the bending point of the exponential relationship that characterises the tube law of collapsible tubes), using least-squares linear regression. In addition, the shape of the velopharynx was assessed by measuring the anteroposterior and bilateral distance at each $\mathrm{Pn}$ level, before and during GG stimulation. As the magnitude of the diameters depended on $P_{n}$, we used for each patient the diameters at the middle of the area $/ P$ n range used for calculation of the slope, and expressed the shape as the ratio of these diameters.

Data are presented as mean \pm SD. The effects of GGa-ES and GGp-ES, as well as comparisons between groups (cephalometric parameters) and between the sites of stimulation, were compared using ANOVA for repeated measures. Correlations were assessed by the least-squares method. Stepwise hierarchical regression analysis (SPSS, Inc., Chicago, IL, USA) was used to test for independent effects of interdependent variables. $\mathrm{p}<0.05$ was considered statistically significant.

\section{RESULTS}

\section{Anthropometric and polysomnographic characteristics}

The anthropometric and polysomnographic characteristics of the study subjects $(n=14$; all male) are given in table 1 . Patients 


\begin{tabular}{|c|c|c|}
\hline TABLE 1 & $\begin{array}{l}\text { polysomn } \\
14 \text { study }\end{array}$ & \\
\hline Characteristics & Mean $\pm S D$ & Range \\
\hline AHI events $\cdot h^{-1}$ & $54.1 \pm 15.9$ & $21-78$ \\
\hline Age yrs & $53.4 \pm 10.5$ & $34-66$ \\
\hline $\mathrm{BMI} \mathbf{k g} \cdot \mathrm{m}^{-2}$ & $32.3 \pm 2.5$ & $28-35$ \\
\hline Apnoeas/total \% & $64.7 \pm 2.7$ & $17-100$ \\
\hline Lowest $\mathrm{Sp}, \mathrm{O}_{2} \%$ & $70.1 \pm 13.4$ & $55-91$ \\
\hline
\end{tabular}

AHI: apnoea-hypopnoea index; apnoeas/total: ratio of apnoeas of all events; $\mathrm{Sp}, \mathrm{O}_{2}$ : arterial oxygen saturation measured by pulse oximetry recorded during the sleep study.

were predominantly middle-aged, overweight males (11 out of 14 with $\mathrm{BMI}>30$ ) and all but one had severe OSA (AHI $>40$ events $\left.\cdot h^{-1}\right)$.

\section{Cephalometry}

The results of the cephalometric measurements, as defined in the methods section, are presented in table 2 . Based on the ratio between the GG and the mouth-box cross-sectional area in the sagittal plane, the patients could be divided arbitrarily into two equal groups $(n=7)$, namely patients with a relatively high $(>0.75)$ and low $(\leqslant 0.75)$ ratio. It can be seen that the higher ration was due primarily to the larger size of the tongue of these patients, as the area of the mouth-box, the angle reflecting the position of the mandible in relation to the maxilla, the distance between the hyoid bone to the mandible, and the size of the pharynx and soft palate, were similar in both groups. Also, the caudal-posterior part of the GG, likely to exert the main protrusive force, tended to be larger in these patients.

\section{Flow}

Figure 2 depicts the effect of GG stimulation in a representative patient. It can be seen that: 1) electrical stimulation shifted the flow $/ P_{n}$ curve to the left, toward lower pressures, as a result of higher flow rates at any given $P_{n} ; 2$ ) the slope of the curves remained nearly unchanged; 3) the effect of GGp-ES was substantially larger than that from GGa-ES. In this patient $P_{\text {crit }}$ decreased from baseline of $5.4 \mathrm{cmH}_{2} \mathrm{O}$ to 2.8 and $-5.6 \mathrm{cmH}_{2} \mathrm{O}$ during GGa-ES and GGp-ES, respectively. Mean data for the whole group are shown in figure 3 . It can be seen that the response to GGa-ES was variable, decreasing $P_{\text {crit }}$ in the majority but not all patients. The mean $P_{\text {crit }}$ decreased during GGa-ES insignificantly from baseline of $3.7 \pm 2.3$ to $2.9 \pm 3.5 \mathrm{cmH}_{2}$. GGp-ES, however, lowered $P$ crit in all subjects, and the mean was $-2.4 \pm 3.7 \mathrm{cmH}_{2} \mathrm{O}(\mathrm{p}<0.001)$. Additional data derived from the flow $/ P$ n relationship measurements are given in table 3 . It can be seen that the change in Pcrit during stimulation was significantly larger with GGp-ES. Similarly, when assessing flow at atmospheric pressure (i.e. without CPAP, $\left.P_{n}=0\right)$, only GGp-ES produced a significant increase in flow rate. This effect occurred because the number of patients with $P_{\text {crit }}<0$ increased from 1 to 10 patients. Both modes of GG stimulation did not affect the slope of flow $/ P \mathrm{n}$, and therefore its reciprocal, upstream resistance, remained unchanged.

\section{Pharyngoscopy}

Pharyngoscopy was performed in 11 of the patients. Figure 4 illustrates the effect of electrical stimulation on velopharyngeal cross-sectional area in one of the patients. It can be seen that: 1) the velopharynx enlarged by electrical stimulation applied at both sites; 2) the effect of GGp-ES was larger than that of GGa-ES at both levels of $P_{n}$, and only GGp-ES opened the occluded velopharynx at $P_{n}=0.3$. GGp-ES enlarged the velopharynx both in the anterior-posterior and lateral direction, while the GGa-ES affected mainly the anterior-posterior direction. For the whole group, the baseline area $/ \mathrm{Pn}_{\mathrm{n}}$ slope (i.e. compliance of the site of collapse, $17.8 \pm 9.8 \mathrm{~mm}^{2} \cdot \mathrm{cmH}_{2} \mathrm{O}^{-1}$ ), remained nearly unchanged during GGa-ES and GGp-ES $\left(19.2 \pm 8.5\right.$ and $15.9 \pm 9.6 \mathrm{~mm}^{2} \cdot \mathrm{cmH}_{2} \mathrm{O}^{-1}$, respectively). Velopharyngeal end-expiratory area, measured at the middle of the area/Pn slope, was $74.5 \pm 38.0 \mathrm{~mm}^{2}$ and increased to $88.2 \pm 40.1$ $(p>0.1)$ and to $130.0 \pm 68.7 \mathrm{~mm}^{2}(p<0.001)$ during GGa-ES and GGp-ES, respectively $((p<0.05)$ for the comparison of the two sites of stimulation). The difference between the two sites of stimulation was due mainly to the difference in their effect on the lateral diameter. As illustrated in figure 5, both GGa-ES and GGp-ES tended to enlarge the anteroposterior (sagittal)

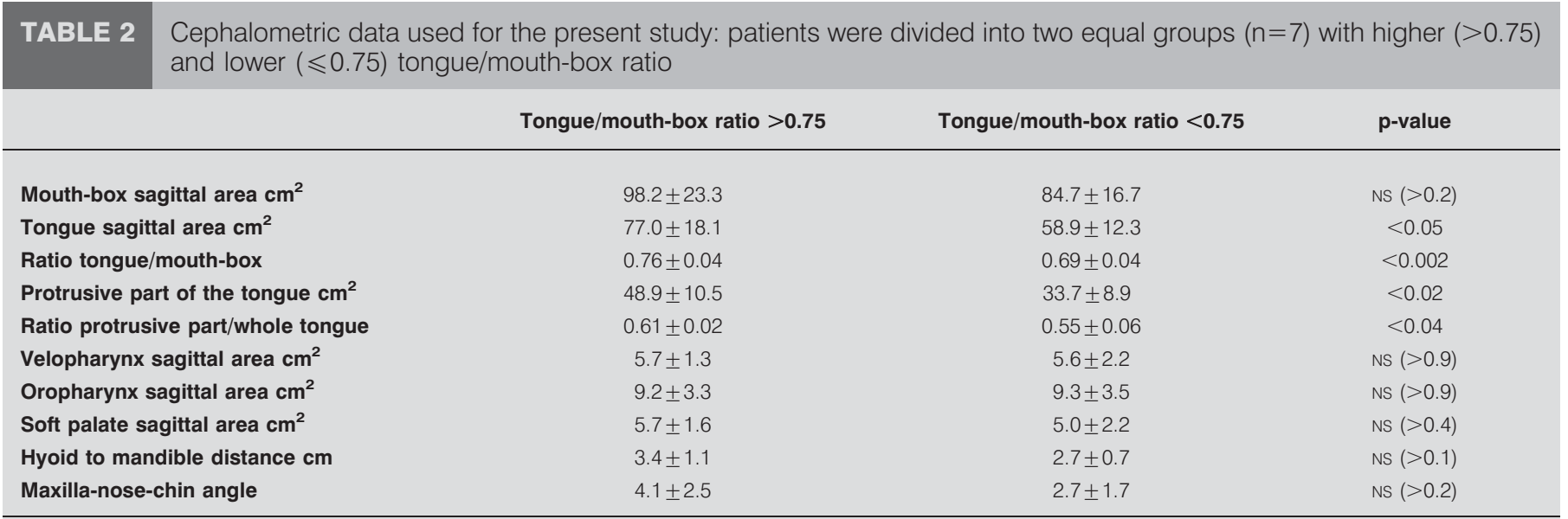

Data are presented as mean \pm SD. NS: not significant. 


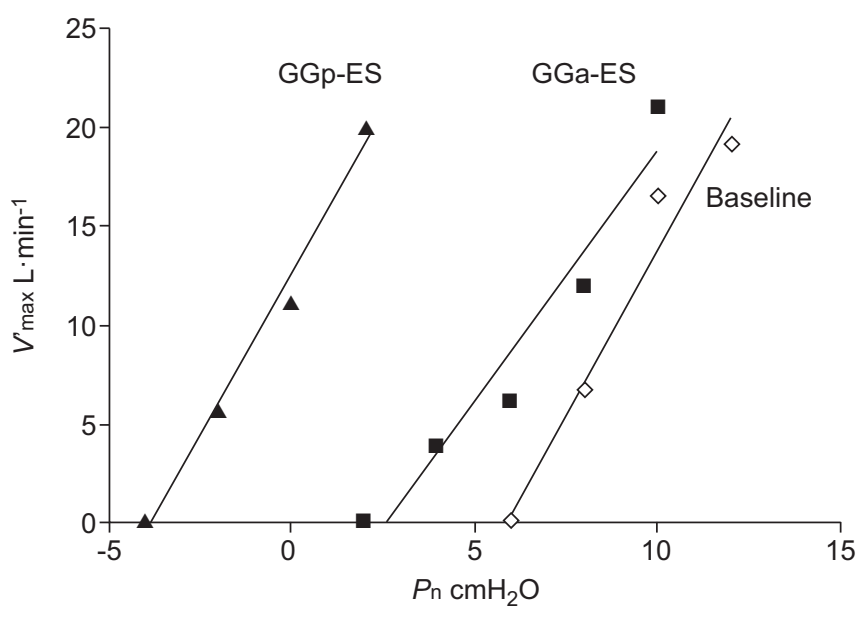

FIGURE 2. Pressure-flow relationship in one study subject at baseline and during electrical stimulation at the anterior (retro-gnathal, GGa-ES) and the posterior (pre-pharyngeal, GGp-ES) sides of the genioglossus. V'max : maximal inspiratory airflow; $P_{n}$ : nasal pressure.

diameter of the velopharynx $(\mathrm{p}=0.078$ and $\mathrm{p}<0.01$, respectively). However, while the lateral diameter remained unchanged with a tendency to decrease during GGa-ES ( $p>0.2$ for comparison with baseline), it increased significantly during GGp-ES ( $\mathrm{p}<0.01)$.

\section{Relationship between baseline parameters and the response to electrical stimulation}

The individual response to electrical stimulation was defined as $\Delta P$ crit. The response to GG stimulation was independent of body weight, age or polysomnographic parameters.

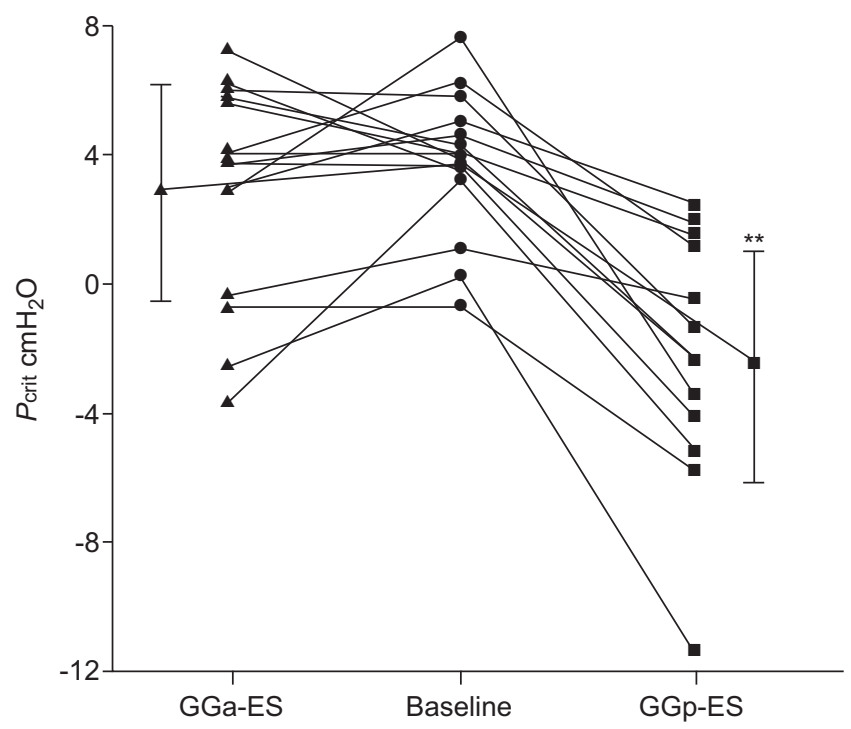

FIGURE 3. Individual and mean \pm SD critical value of end-expiratory pressure (Pcrit) values for all patients at baseline and during electrical stimulation of the genioglossus at the anterior (GGa-ES) and posterior (GGp-ES) area of the genioglossus. ${ }^{* *}$ : $p<0.01$ for comparison of GGp-ES with baseline and GGa-ES.

\begin{tabular}{|c|c|c|c|c|}
\hline \multirow[t]{2}{*}{ TABLE 3} & \multicolumn{4}{|c|}{$\begin{array}{l}\text { Data derived from the pressure-flow relationship } \\
\text { before and during electrical stimulation at the } \\
\text { anterior (GGa-ES) and posterior (GGp-ES) areas } \\
\text { of the genioglossus }\end{array}$} \\
\hline & & Baseline & GGa-ES & GGp-ES \\
\hline \multicolumn{2}{|l|}{ Pcrit $\mathrm{cmH}_{2} \mathrm{O}$} & $3.7 \pm 2.3$ & $2.9 \pm 3.5$ & $-2.4 \pm 3.7^{\star \star}$ \\
\hline \multicolumn{2}{|c|}{$\Delta P_{\text {crit }} \mathrm{cmH}_{2} \mathrm{O}$} & & $0.82 \pm 2.8$ & $6.1 \pm 2.9^{\star \star}$ \\
\hline \multicolumn{2}{|c|}{ Rus $\mathrm{cmH}_{2} \mathrm{O} \cdot \mathrm{L}^{-1} \cdot \mathrm{s}^{-1}$} & $28.5 \pm 12.2$ & $27.2 \pm 13.6$ & $28.4 \pm 16.0$ \\
\hline \multicolumn{2}{|c|}{ Flow at $P_{n}=0 \mathrm{~L} \cdot \mathrm{min}^{-1}$} & $0.02 \pm 0.07$ & $1.1 \pm 2.4$ & $6.0 \pm 5.6^{* *}$ \\
\hline
\end{tabular}

\section{Relationship between cephalometric parameters and the} response to electrical stimulation

Out of the large number of cephalometric parameters described in previous studies [9-11], including the seven parameters we considered as likely to affect the response to contraction of the GG (fig. 1), only the sagittal area of the tongue (corrected for the size of the bony walls of the mouth cavity) correlated significantly $(\mathrm{R}=0.63, \mathrm{p}<0.02)$ with $\Delta P_{\text {crit }}$ during GGp-ES (fig. 6). Patients with large tongue relative to the oral cavity (tongue/mouth-box ratio $>0.75, \mathrm{n}=7$ ) had a mean $\Delta P_{\text {crit }}$ of $8.0 \pm 2.2 \mathrm{cmH}_{2} \mathrm{O}$, compared with patients with smaller tongue/mouth-box ratio $\left(\Delta P\right.$ crit $3.8 \pm 2.1 \mathrm{cmH}_{2} \mathrm{O}$, $\mathrm{p}<0.005)$. However, we found no correlation between $\Delta P_{\text {crit }}$ during GGa-ES and the above parameters.

\section{Relationship between flow parameters and the response to electrical stimulation}

Baseline $P_{\text {crit }}$ and upstream resistance did not correlate with $P_{\text {crit }}$ during GGp-ES, and the latter was determined primarily by $\Delta P_{\text {crit }}(\mathrm{R}=0.78, \mathrm{p}<0.01)$. Therefore, although one may expect low $P_{\text {crit }}$ to contribute to the magnitude of $V^{\prime}$ max at atmospheric pressure obtained during electrical stimulation, the correlation between these variables was not significant $(R=-0.31)$. However, $V^{\prime} \max$ at atmospheric pressure during GGp-ES correlated significantly with both $P$ crit during stimulation and $\Delta P_{\text {crit }}(\mathrm{R}=-0.73$ and $\mathrm{R}=0.69$, respectively, $\mathrm{p}<0.01$ for both).

\section{Relationship between velopharynx shape and the response to electrical stimulation}

$\Delta P$ crit was affected also by the baseline shape of the velopharynx (fig. 7): a flat-elliptical shape of the velopharyngeal orifice with low anterior-posterior to lateral diameter ratio tended to have a larger response to GGp-ES $(R=-0.74, p<0.01)$. However, the anteroposterior to lateral diameter ratio was also closely and inversely related to the sagittal size of the tongue $(R=-0.61$, $\mathrm{p}<0.05$ ), with large tongues associated with narrow pharynx. In contrast, we found no correlation between $\Delta P$ crit during GGa-ES and the above parameters. Also, no correlation was found between $\Delta P_{\text {crit }}$ and the compliance at the site of collapse.

\section{DISCUSSION}

The present study evaluated the effects of electrically induced contraction of the main pharyngeal dilator, the GG, on flow-mechanics 


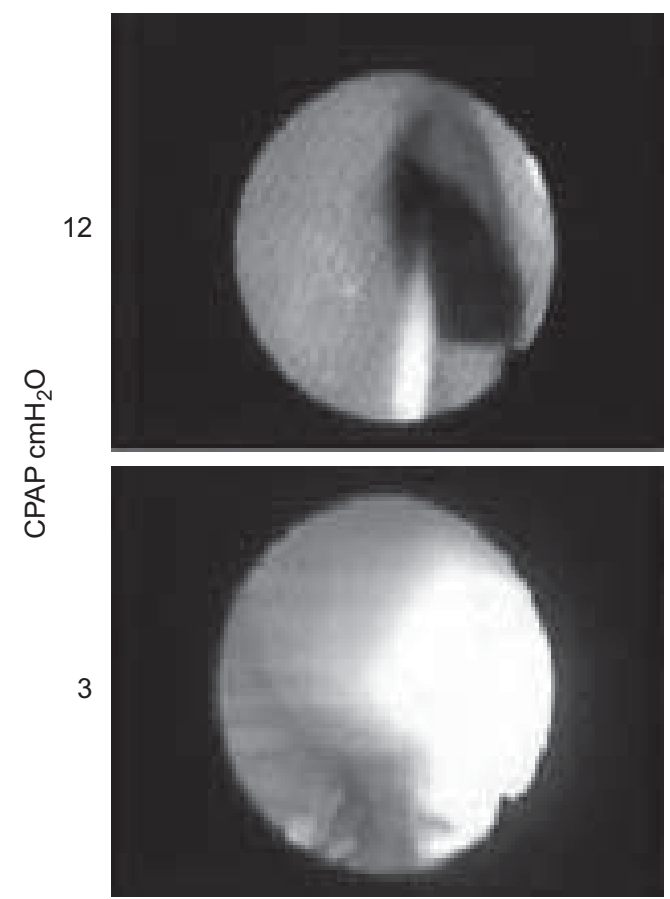

Baseline
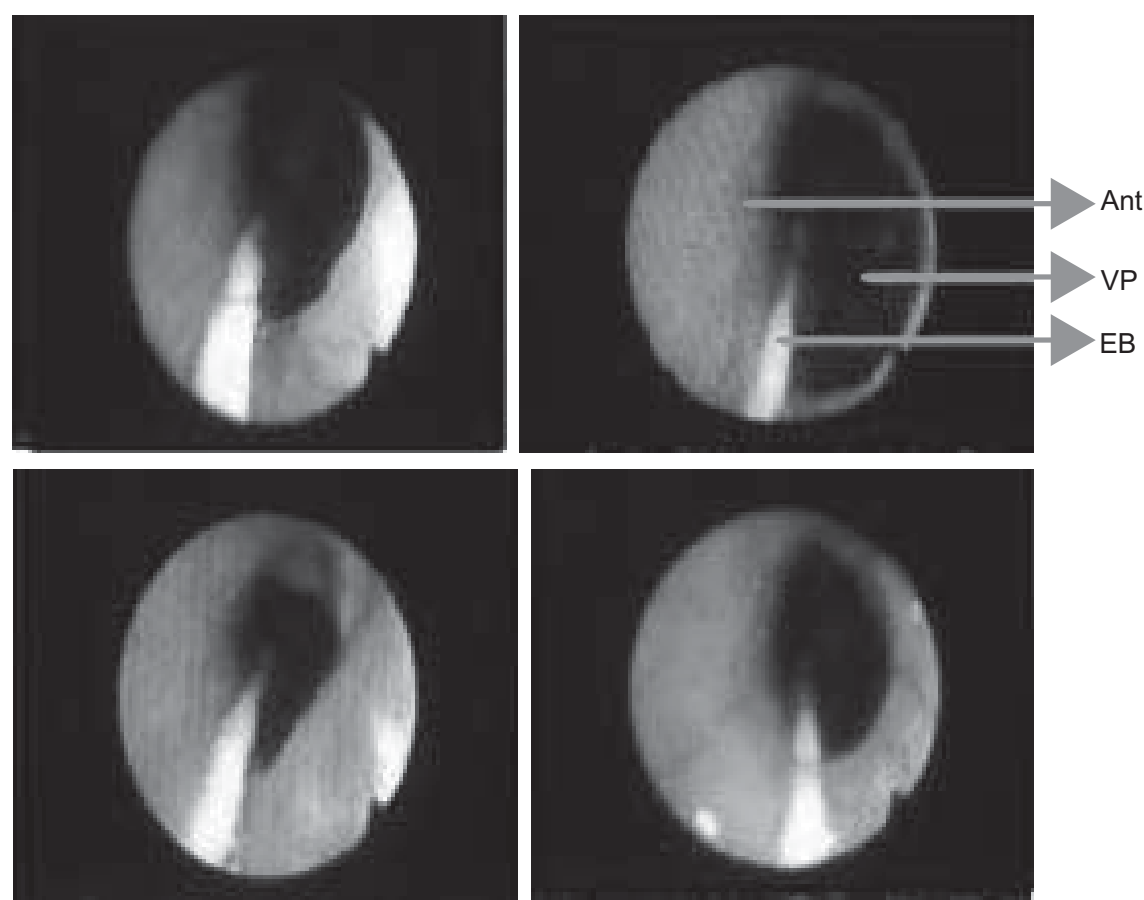

GGa-ES

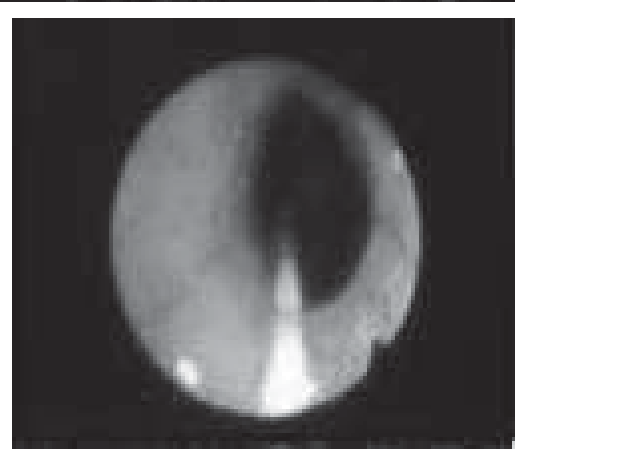

GGp-ES

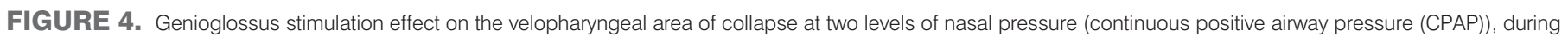
anterior (GGa-ES) and posterior (GGp-ES) electrical stimulation. Ant: anterior; VP: velopharynx; EB: oesophageal balloon tube.

and pharyngeal patency in patients with OSA. Continuing our previous work [7], we now assessed the effects of the site of stimulation and the importance of cephalometric parameters. We found that both the specific area of the GG recruited and specific patient characteristics are important: stimulation of the posterior part of the GG stabilised the pharynx more than the anterior part, and the best results were obtained in patients with a large tongue and narrow pharynx.

Previous studies using intramuscular stimulating electrodes to assess the effect of electrical stimulation of the GG on pharyngeal patency usually addressed this muscle as acting
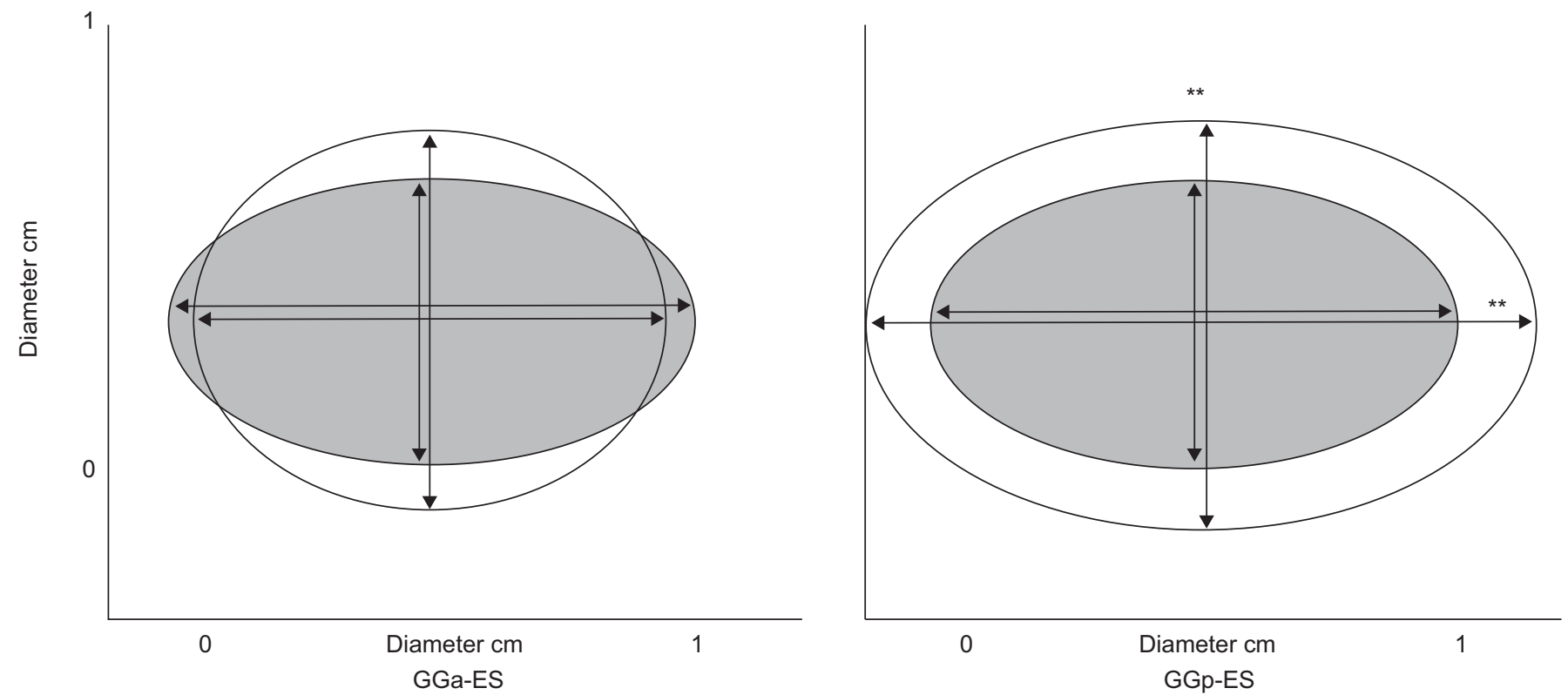

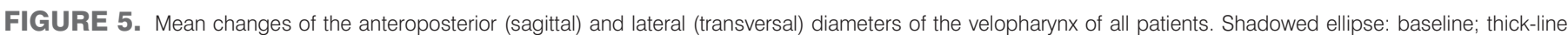

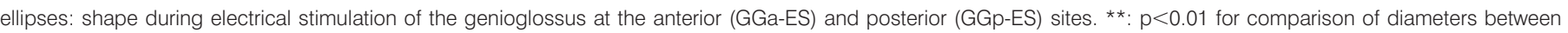
electrical stimulation and baseline. The increase in sagittal diameter with GGa-ES was of borderline significance $(p=0.078)$. 


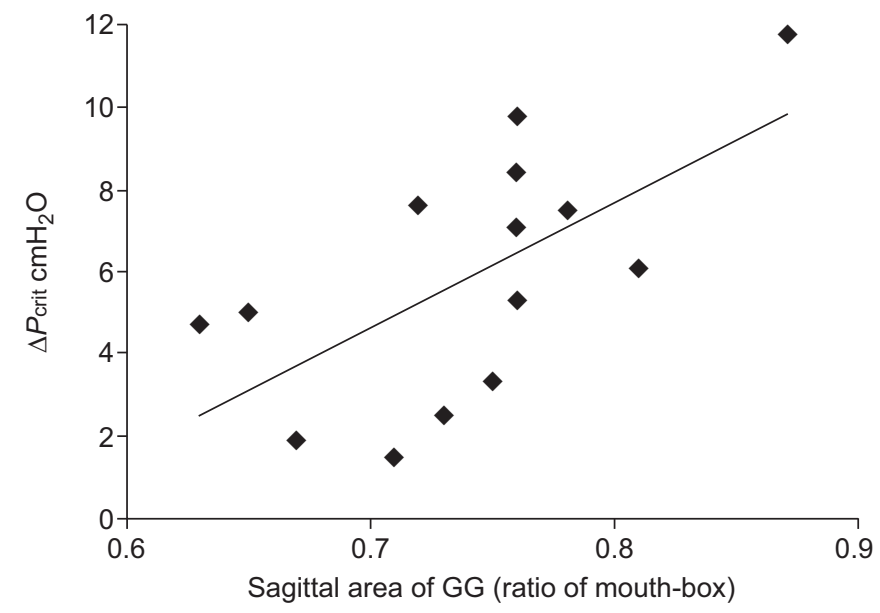

FIGURE 6. Relationship between the size of the genioglossus (GG) assessed as its sagittal cross-sectional area and given as a ratio of the sagittal cross-sectional area of the mouth-box, and the change in the critical value of end-expiratory pressure ( $P_{\text {crit) }}\left(\Delta P_{\text {crit, }}\right.$ baseline $P_{\text {crit }}-P_{\text {crit }}$ during $G G$ contraction). The response to electrical stimulation was larger with increasing size of the tongue. $R=0.632, p<0.02$.

mechanically as a single functional unit and used one stimulation site [6, 7, 14, 15]. However, the GG fibres are arranged as a hand held fan, and the effects of contraction of the GG may be understood by considering the direction of the fibres [16]: the horizontal-longitudinal fibres are the actual tongue protrusors that draw the tongue forward, while the anterior fibres are oriented vertically, and their contraction, also when acting together with the longitudinal fibres, act to depress and draw down the tongue [8]. We previously observed that contraction of the depressor part of the GG may sometimes obstruct the pharynx, by causing posterior bulging of the dorsal part of the tongue (like squeezing a ball) [16]. Therefore, we hypothesised that stimulation of different areas of the GG affects its vector of contraction, and targeting the main body of the protrusive part of the GG may have a larger effect on pharyngeal patency, diminishing recruitment of vertical GG fibres co-activated by anterior placed electrodes (fig. 1). The present findings seem to confirm this assumption, indicating that stimulation of specific areas of the tongue is needed to obtain an optimal flow-mechanical effect.

An additional finding of the present study was the interrelationship between velopharyngeal shape, tongue size and the response to GG contraction. It has been previously shown by several (although not all) studies that patients with OSA tend to have upper airways with a high anteroposterior/lateral diameter ratio, suggesting that the shape of the pharynx may have an independent pathophysiological significance [17-20]. It has been postulated that an anteroposteriorly oriented elliptical shape may be disadvantageous and predispose the pharynx to collapse, as the GG that acts primarily to enlarge the pharynx anteriorly is likely to be less effective if the pharyngeal shape is oriented in the anteroposterior direction [18]. This intuitive hypothesis was confirmed by the significant correlation between shape and response to GG stimulation observed in our patients. However, the explanation for this finding seems to be complex. We found that the anteroposterior/lateral diameter ratio was inversely related to the size of

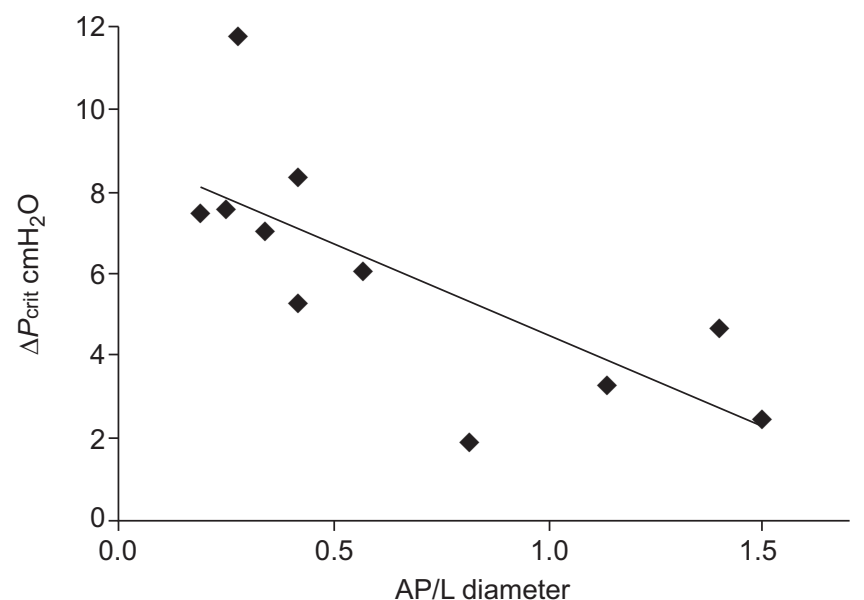

FIGURE 7. Relationship between the shape of the velopharynx and the response to electrical stimulation of the genioglossus (posterior site). The shape is characterised by the ratio of anteroposterior to lateral (AP/L) diameters. The response to stimulation is given by the change in the critical value of end-expiratory pressure ( $\Delta P_{\text {crit) }}$ during stimulation. The response to electrical stimulation was larger in patients with a narrow anteroposterior and large lateral diameter. $R=-0.738, p<0.01$.

the tongue, probably because a large tongue is likely to compress the pharynx. Although we corrected the size of tongue to that of the bony structure of the mouth cavity, a similar correlation with the shape was found also with the uncorrected measured size of the tongue. Accordingly, the larger mechanical effect could be related to either the size of the tongue and/or to the shape of the velopharynx. We found a difference in change in shape produced by GGp-ES and GGaES: both sites of stimulation increased the anteroposterior diameter of the velopharynx, but the superiority of GGp-ES appeared to be due to its additional enlarging effect on the lateral diameter (fig. 5).

Most probably, the effects of GG contraction (and probably additionally co-activated tongue muscles) on the velopharynx includes forces additive to unloading the weight of the tongue [21]. Electrical stimulation of the GG appears to affect also the lateral pharyngeal walls, probably involving mechanical coupling of the base of tongue and the soft palate via the fauces [22]. Using magnetic resonance imaging (MRI) with a novel spatial modulation of magnetisation (SPAMM) technique, a significant stretch of lateral tissue was observed during medial hypoglossus branch stimulation [23]. Although in this study, performed in rats, lateral enlargement was observed only in the oropharyngeal region, significant enlargement in the lateral diameter was observed also at the level of the velopharynx during stimulation of the medial, lateral and whole hypoglossus in cats [24]. As soft tissue is not compressible, contraction of the GG probably enabled enlargement of the pharynx by caudal displacement of soft tissue outside the maxilo-mandibular bony enclosure, as described by TSUIKI et al. [11]. While compliance may decrease at the level of the oropharynx during GG stimulation [15], we did not find a change in compliance at the level of the velopharynx, confirming previous observations in humans [7, 15], as well as in animals studied with MRI $[14,25]$. This finding supports the notion that GG stimulation 
alters velopharyngeal patency primarily by its effect on the surrounding external pressure [26].

Several limitations of this study and potential confounders have to be acknowledged. First, this is a laboratory-based study, evaluating physiological parameters under propofol anaesthesia. We chose to use anaesthesia to assess the mechanical effect of electrical stimulation and to enable endoscopic evaluation over a wide range of $P$ n levels, a task that could not be performed during normal sleep. Both propofol and isoflurane are being used to evaluate pharyngeal mechanics during anaesthesia and were found to cause a dosedependent increase in pharyngeal collapsibility [27]. As during sleep, both anaesthetics abolish GG reflex activation, while enabling spontaneous breathing and their use was often referred to as "drug-induced sleep" [28]. Nevertheless, the use of propofol poses limitations on the extrapolation of our findings to conditions occurring during sleep. Propofol may produce more muscle relaxation than sleep rendering the upper airway more passive and more collapsible [29]. However, this only means that we may expect baseline $P_{\text {crit }}$ of our patients to be lower (i.e. the velopharynx more stable) during sleep. Propofol does not influence involuntary isometric skeletal muscle strength [30, 31], and the magnitude of stimulation-induced change in Pcrit during sleep and propofol anaesthesia is similar [32]. Therefore, we believe that the experimental conditions actually underestimated the expected flow response to GG stimulation at atmospheric pressure. In our patients, 13 out of 14 were completely obstructed at atmospheric pressure, and GGp-ES enabled flow at $P_{\mathrm{n}}=0$ in 10 patients. With lower baseline Pcrit expected during sleep, and equal $\Delta P$ crit, a larger clinically relevant effect of GGp-ES may be anticipated, i.e. GGp-ES is likely to enable higher flow rates at ambient pressure during sleep than during anaesthesia. Although reductions in $P_{\text {crit }}$ were found to be similar with GG and hypoglossus stimulation during sleep [6], our results indicate that specific areas of the tongue should be stimulated to improve pharyngeal dilatation. As intramuscular electrodes also stimulate sensory nerve fibres and cause arousal, hypoglossus nerve stimulation may enable painless tongue muscle contraction. However, it is unclear to what extent selective stimulation is feasible with neural stimulation. The hypoglossus can be divided into medial and lateral branches, with the former innervating the GG, as well as the geniohyoid and intrinsic tongue muscle $[13,14,23]$. The intramuscular branching of the hypoglossus has not been evaluated. Therefore, correlation between responses to GG and hypoglossus stimulation can be assessed only after an implantable device producing effective hypoglossus nerve stimulation is developed. The line drawn in figure 1 to separate the predominantly protrusor from the predominantly depressor part of the GG is rather schematic, as the GG is not divided into distinct functional units. This may be the cause for the close relationship between the depressor, protrusor and total size of the tongue that precluded demonstration of an independent relationship between the protrusive part of the tongue and the response to GG stimulation. Therefore, the improved response observed could be attributed to either unloading a heavier tongue, or contraction of a larger protrusive muscle (table 2), or both. It should be noted that in the present study, as in our previous study performed under propofol sedation, the primary site of collapse was the velopharynx (or combined velo- and oropharynx) in all patients [7]. Accordingly, as we have previously found that the effect of GG stimulation on the oropharynx is larger than on the velopharynx [7], a larger effect is expected in patients with the oropharyngeal as the primary site of obstruction. The difference in response to the two sites of stimulation could also be due to differences in co-activation of retractor tongue muscles, but retractor recruitment is unlikely to produce an important change in response [16]. We did not evaluate systematically the reproducibility of our results, but current and previous experience based on insertion of additional electrodes in the same area of stimulation suggests that the effects of stimulation are closely reproducible. Small changes in the level of anaesthesia, as well as head or mandibular position known to affect the response to GG contraction [33], could occur between the evaluations of the two sites of stimulation, despite our efforts to maintain stable conditions. However, the two sites were evaluated in random order to prevent systematic error. Measurement of the velopharyngeal area could be distorted by axial movements of the pharynx during stimulation, changing the distance between the pharyngoscope and the measured region [13]. However, no axial movements occurred during stimulation, as opposed to substantial axial shift during inspiration, as clearly evident from the relationships between the endoscope, pharyngeal structures and the oesophageal tube that was marked at regular intervals. It should be noted that the site of collapse was determined endoscopically in conjunction with flow monitoring during gradual reduction of CPAP, enabling accurate assessment of the primary area responsible for pharyngeal occlusion. We used the cross-sectional area to pressure relationship as an estimate of the compliance of the velopharyngeal site of collapse, although compliance is, per definition, the relationship between volume and pressure. This approach was used in most studies evaluating regional compliance of the pharynx $[7,14,15$, $16,23]$, and may be more relevant for the assessment of pharyngeal flow mechanics than the compliance of the whole upper airway. Finally, we limited baseline anatomic evaluation to simple cephalometry, considered sufficient for the information needed for the present study. Several new and more sophisticated modalities based on three-dimensional reconstruction of computed tomography (CT) and MRI imaging of the upper airway have been recently developed [34, 35], that enable much more accurate anatomic and morphologic characterisation of the pharynx and peripharyngeal structures, and may be also useful for the evaluation of muscle stimulation [36].

The need for a specific site of GG activation combined with specific anatomic characteristics to achieve effective pharyngeal dilatation reflects the well-known interactions between neuromuscular and structural/anatomic factors in the pathogenesis of OSA [37]. Functional electrical stimulation of striated muscles provides an important tool to assess their physiological effect and has been largely employed for the study of the mechanical effects of upper airway dilator muscles both in animals and humans. In addition, however, evaluation of the effect of stimulation of the main tongue protrusor, the GG, provides information that may be useful for future therapeutic interventions. The main treatment modality for OSA is the application of CPAP, a remedy that is poorly tolerated, leading to low compliance or frank refusal by many 
patients. Considering the obvious role of neuromuscular mechanisms in the pathogenesis of OSA, since apnoeas occur only during sleep in association with a decline in GG activity [38], it is reasonable to assume that adequately applied electrical activation of this muscle may prevent pharyngeal collapse. Therefore, attempts to stimulate the GG in OSA patients for therapeutic purposes have been undertaken ever since the physiological importance of this muscles' action was appreciated, with variable results [2, 36, 39, 40]. A major advance has been achieved recently when a group of OSA patients were implanted with chronic hypoglossus nerve stimulators, using cuff or sleeve-like electrodes implanted unilaterally around a hypoglossus nerve, with stimulation triggered by inspiration [3, 41]. Although technical malfunctions precluded prolonged follow-up, large decreases in apnoeas and hypopnoeas were observed in most patients. These encouraging findings triggered ongoing attempts to improve the new device. Consequently, the topics evaluated in the present study are clinically important, as our findings provide useful information relevant for hypoglossus stimulation and selection of patients most likely to benefit from this new treatment modality. If the response to GG stimulation is found in prospective studies to correlate with the response to hypoglossus stimulation, the former may be used for screening OSA patients expected to have a good response to hypoglossus stimulation. It should be noted that no complications from electrode insertion occurred during our studies. Considering the instability of pharyngeal shape, which is highly dependent on the level of $P n$, and the relative difficulty obtaining this parameter, measuring the size of the tongue using radiography (or CT) and relating it to the size of the bony cage of the mouth cavity may be a simpler alternative. Our findings suggest that the best response to electrical stimulation of the tongue is expected to be obtained in OSA patients with large tongues, during preferential activation of the longitudinal (protrusive) fibres of the GG. However, considering the scatter observed in figures 6 and 7, additional parameters that will improve patient selection are desirable.

\section{STATEMENT OF INTEREST}

A statement of interest for the present study can be found at www.erj. ersjournals.com/site/misc/statements.xhtml

\section{REFERENCES}

1 Young T, Palta M, Dempsey J, et al. The occurrence of sleepdisordered breathing among middle-aged adults. $N$ Engl J Med 1993; 328: 1230-1235.

2 Miki H, Hida W, Shindoh C, et al. Effects of electrical stimulation of the genioglossus on upper airway on airway resistance in anesthetized dogs. Am Rev Respir Dis 1989; 140: 1279-1284.

3 Schwartz AR, Bennett ML, Smith PL, et al. Therapeutic electrical stimulation of the hypoglossal nerve in obstructive sleep apnea. Arch Otolaryngol Head Neck Surg 2001; 127: 1216-1223.

4 Odeh M, Schnall R, Gavriely N, et al. Effect of upper airway muscle contraction on supraglottic resistance and stability. Respir Physiol 1993; 92: 139-150.

5 Schnall RP, Pillar G, Kelsen SG, et al. Dilatory effects of upper airway muscle contraction induced by electrical stimulation in awake humans. J Appl Physiol 1995; 786: 1950-1956.

6 Oliven A, O'Hearn DJ, Boudewyns A, et al. Upper airway response to electrical stimulation of the genioglossus in obstructive sleep apnoea. J Appl Physiol 2003; 95: 2023-2029.
7 Oliven A, Tov N, Geitini L, et al. Effect of genioglossus contraction on pharyngeal lumen and airflow in sleep apnoea patients. Eur Respir J 2007; 30: 748-758.

8 Gray H. Anatomy of the Human Body. 20th Edn. New York, Bartleby.com, 2000; pp. 1129-1131.

9 Brander PE, Mortimore IL, Douglas NJ. Effect of obesity and erect/supine posture on lateral cephalometry: relationship to sleep-disordered breathing. Eur Respir J 1999; 13: 398-402.

10 Xiujun Y, Keisaku F, Kazuhisa U, et al. Cephalometric analysis in obese and nonobese patients with obstructive sleep apnea. Chest 2003; 124: 212-218.

11 Tsuiki S, Isono S, Ishikawa T, et al. Anatomical balance of the upper airway and obstructive sleep apnea. Anesthesiology 2008; 108: 1009-1015.

12 Stone M. A guide to analysing tongue motion from ultrasound images. Clin Linguist Phon 2005; 19: 455-501.

13 Kuna ST. Effects of pharyngeal muscle activation on airway size and configuration. Am J Respir Crit Care Med 2001; 164: 1236-1241.

14 Brennick MJ, Gefter WB, Margulies SS. Mechanical effects of genioglossus muscle stimulation on the pharyngeal airway by MRI in cats. Respir Physiol Neurobiol 2007; 156: 154-164.

15 Isono S, Tanaka A, Nishino T. Effects of tongue electrical stimulation on pharyngeal mechanics in anaesthetized patients with obstructive sleep apnoea. Eur Respir J 1999; 14: 1258-1265.

16 Oliven A, Odeh M, Geitini L, et al. Effect of coactivation of tongue protrusor and retractor muscles on pharyngeal lumen and airflow in sleep apnea patient. J Appl Physiol 2007; 103: 1662-1668.

17 Rodenstein DO, Dooms G, Thomas Y, et al. Pharyngeal shape and dimensions in healthy subjects, snorers and patients with obstructive sleep apnoea. Thorax 1990; 45: 722-727.

18 Leiter JC. Upper airway shape: is it important in the pathogenesis of obstructive sleep apnea? Am J Respir Crit Care Med 1996; 153: 894-898.

19 Schwab RJ, Pasirstein M, Pierson R, et al. Identification of upper airway anatomic risk factors for obstructive sleep apnea with volumetric magnetic resonance imaging. Am J Respir Crit Care Med 2003; 168: 522-530.

20 Walsh JH, Leigh MS, Paduch A, et al. Evaluation of pharyngeal shape and size using anatomical optical coherence tomography in individuals with and without obstructive sleep apnoea. J Sleep Research 2008; 17: 230-238.

21 Isono S, Atsuko T, Takashi N. Dynamic interaction between the tongue and soft palate during obstructive apnea in anesthetized patients with sleep disordered breathing. J Appl Physiol 2003; 95: 2257-2264.

22 Isono S, Tanaka A, Sho Y, et al. Advancement of the mandible improves velopharyngeal airway patency. J Appl Physiol 1995; 79: 2132-2138.

23 Brennick MJ, Pickup S, Dougherty L, et al. Pharyngeal airway wall mechanics using tagged magnetic resonance imaging during medial hypoglossal nerve stimulation in rats. J Physiol 2004; 561: 597-610.

24 Kuna ST. Regional effects of selective pharyngeal muscle activation on airway shape. Am J Respir Crit Care Med 2004; 169: 1063-1069.

25 Van Zutphen C, Janssen P, Hassan M, et al. Regional velopharyngeal compliance in the rat: influence of tongue muscle contraction. NMR Biomed 2007; 20: 682-691.

26 Oliven A, Kaufman E, Kaynan R, et al. Mechanical parameters determining pharyngeal collapsibility in patients with sleep apnea. J Appl Physiol 2010; 109: 1037-1044.

27 Eikermann M, Malhotra A, Fassbender P, et al. Differential effects of isoflurane and propofol on upper airway dilator muscle activity and breathing. Anesthesiology 2008; 108: 897-906.

28 Boudewyns A, Marklund M, Hochbab W. Alternatives for OSAHS treatment: selection of patients for upper airway surgery and oral appliances. Eur Respir Rev 2007; 16: 132-145. 
29 Eastwood PR, Platt PR, Shepherd K, et al. Collapsibility of the upper airway at different concentrations of propofol anesthesia. Anesthesiology 2005; 103: 453-454.

30 Nishina K, Mikawa K, Kodama S, et al. The effects of enflurane, isoflurane, and intravenous anesthetics on rat diaphragmatic function and fatigability. Anesth Analg 2003; 96: 1674-1678.

31 Ginz HF, Zorzato F, Iaizzo PA, et al. Effect of three anaesthetic techniques on isometric skeletal muscle strength. Br J Anaesth 2004; 92: 367-372.

32 Oliven $\mathrm{A}$, Tov $\mathrm{N}$, Odeh $\mathrm{M}$, et al. Electrical stimulation of the genioglossus to improve pharyngeal patency in obstructive sleep apnea: comparison of results obtained during sleep and anesthesia. Harefuah 2009; 148: 315-319.

33 Oliven R, Tov N, Odeh M, et al. Interacting effects of genioglossus stimulation and mandibular advancement in sleep apnea. J Appl Physiol 2009; 106: 1668-1673.

34 De Backer JW, Vos WG, Verhulst SL, et al. Novel imaging techniques using computer methods for the evaluation of the upper airway in patients with sleep-disordered breathing: a comprehensive review. Sleep Med Rev 2008; 12: 437-447.
35 Schwab RJ, Goldberg AN. Upper airway assessment: radiographic and other imaging techniques. Otolaryngol Clin North Am 1998; 31: 931-968.

36 Oliven A, Schnall RP, Pillar G, et al. Sublingual electrical stimulation of the tongue during wakefulness and sleep. Respir Physiol 2001; 127: 217-226.

37 Younes M. Contributions of upper airway mechanics and control mechanisms to severity of obstructive apnea. Am J Respir Crit Care Med 2003; 168: 645-658.

38 Jordan AS, Wellman A, Heinzer RC, et al. Mechanisms used to restore ventilation after partial upper airway collapse during sleep in humans. Thorax 2007; 62: 861-867.

39 Guilleminault C, Powell N, Bowman B, et al. The effect of electrical stimulation on obstructive sleep apnea syndrome. Chest 1995; 107: 67-73.

40 Decker M, Haaga J, Arnold L, et al. Functional electrical stimulation and respiration during sleep. J Appl Physiol 1992; 75: 1053-1061.

41 Kezirian EJ, Boudewyns A, Eisele DW, et al. Electrical stimulation of the hypoglossal nerve in the treatment of obstructive sleep apnea. Sleep Med Rev 2010; 14: 299-305. 